\title{
Distribution of plasminogen activator inhibitor in normal liver, cirrhotic liver, and liver with
} metastases

\author{
P Fitch, B Bennett, N A Booth, A Croll, S W B Ewen
}

\begin{abstract}
Aims-To examine the distribution of PAI-1 antigen in normal and cirrhotic liver and liver with metastases.

Methods-Sections of normal and cirrhotic liver and liver with metastases were stained using the alkaline phosphatase antialkaline phosphatase (APAAP) technique and monoclonal antibody specific for plasminogen activator inhibitor (PAI-1).

Results-PAI-1 antigen was identified as discrete granules in the cytoplasm of hepatocytes in normal liver, particularly around portal tracts and central veins of the liver lobule. In cirrhotic liver a striking reduction of PAI-1 antigen was noted. In liver with metastases increased amounts of PAI-1 antigen were concentrated in hepatocytes around the margins of malignant deposits.

Conclusions-Cirrhotic liver contains considerably less PAI-1 antigen than does normal liver, despite raised plasma concentrations of PAI-1. This may reflect release of hepatic PAI-1 into the circulation or decreased clearance of PAI-1 from the plasma. Secondary malignant deposits in the liver seem to stimulate production of PAI-1 in adjacent hepatocytes. This may influence the invasive process and may contribute to the thrombotic tendency associated with malignancy.
\end{abstract}

(F Clin Pathol 1994;47:218-221)

Tissue plasminogen activator (t-PA) is the principal circulating activator of the fibrinolytic system. t-PA is absorbed along with plasminogen on to fibrin; thus juxtaposed, they generate plasmin, which lyses the fibrin deposit.

Control of t-PA in the circulation is secured by two main mechanisms. Firstly t-PA is inhibited directly and, secondly, it is cleared from the circulation by the liver. In both processes plasminogen activator inhibitor (PAI-1) has an important role. It is the primary plasma protein inhibiting t-PA, other inhibitors such as C1-esterase inhibitor and $\alpha_{2}$ macroglobulin having a secondary role. ${ }^{1} \mathrm{PAI}-1$ also participates in clearance of t-PA by the liver; t-PA in complex with PAI-1 clears more rapidly than free t-PA. ${ }^{2}$ A specific hepatic receptor for t-PA-PAI-1 complex has been shown, ${ }^{3}$ in addition to the mannose and galac- tose receptors that clear free t-PA. ${ }^{4-8}$

The liver contains PAI- $1^{9}$ and hepatocytes in culture release PAI-1. ${ }^{10}$ In situ hybridisation using a PAI-1 probe failed to show PAI-1 mRNA in rat hepatocytes, " but PAI-1 mRNA has been shown in rat liver regenerating after injury to the liver. ${ }^{12} 13$ The role of hepatic PAI1 is presently undefined and its relation to plasma PAI-1 and direct inhibition of t-PA or to clearance of t-PA from plasma remains to be established. The location and distribution of PAI-1 in normal liver is not known.

In hepatic cirrhosis "overall plasma fibrinolytic activity" is increased suggesting that $t-$ PA concentrations are raised. ${ }^{14-16}$ t-PA and PAI-1 antigen concentrations are in fact both strikingly raised in cirrhosis, ${ }^{17} 18$ but these observations do not distinguish between free (active) or complexed (inactive) forms of these molecules. The enhanced fibrinolytic activity in cirrhosis has been shown to be due in part to reduced clearance of t-PA. ${ }^{15}$ Patients with disseminated carcinoma frequently have reduced plasma fibrinolytic activity and raised plasma concentrations of PAI-1. ${ }^{19} 20$ Such patients additionally show a reduced fibrinolytic response to stimuli such as nicotinic acid. ${ }^{15}$

\section{Methods}

Liver sections fixed in $10 \%$ neutral buffered formalin and $4 \mu \mathrm{m}$ in thickness were obtained from the pathology department of the University of Aberdeen. In addition, portions of fresh liver biopsy specimens were obtained before fixation. Normal liver and liver affected by alcoholic cirrhosis $(n=10)$, primary biliary cirrhosis $(n=5)$, metastatic carcinoma $(n=10)$, and primary hepatocellular carcinoma $(n=5)$ were studied. The diagnoses were based on the biopsy result and the results of liver function tests at the time of biopsy.

Tissue sections were stained using the APAAP technique, which was essentially the method described by Cordell et al. ${ }^{21}$ The primary antibody, mouse antibody to human PAI-1 monoclonal antibody, ${ }^{22}$ was applied to the sections for 1 hour in a moist chamber at room temperature. Optimal dilutions of antibody were found by experiment to be 1 in 200 and 1 in 400 . Control sections were stained with a monoclonal antibody of the same subclass $\left(\mathrm{IgG}_{1}\right)$, raised against human neurofilament protein. This antibody was used at a dilution of 1 in 10.

The slides were then washed in $50 \mathrm{mM}$ TRIS-buffered saline (TBS) $(\mathrm{pH} 7 \cdot 6)$, and 

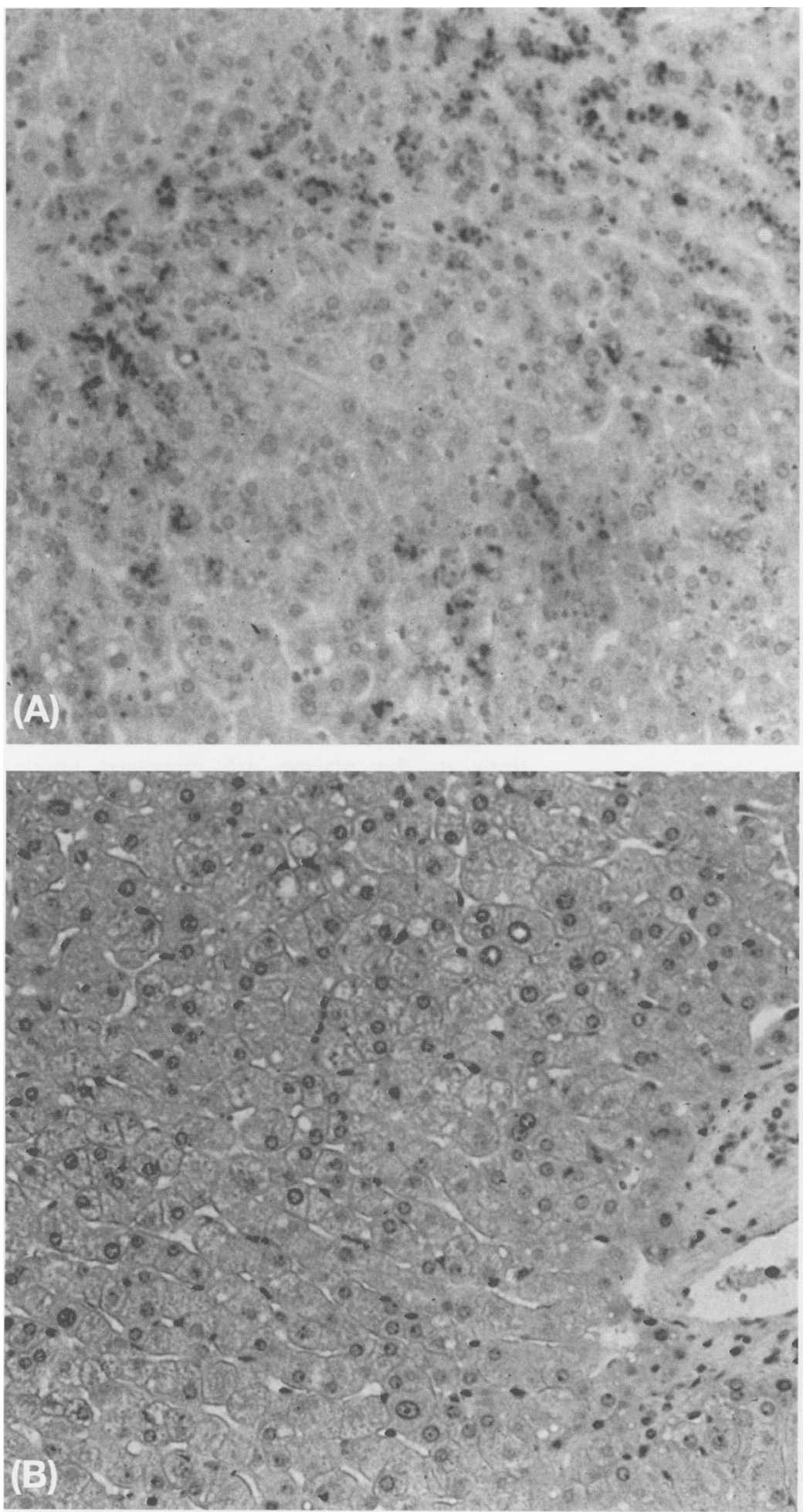

Figure 1 Normal liver section stained with $(A)$ monoclonal antibody to PAI-1; and (B) control antibody. acetate, $\mathrm{pH} 9 \cdot 2$ ) in the order given above. After further washing slides were counterstained in Harris' haematoxylin for 5 seconds and mounted.

The stained liver sections were graded for amount of specific staining of PAI-1 antigen by an experienced pathologist who was not involved in the project and was unaware of the purpose of the study. Each slide was assigned a grade between -, which signified no PAI-1 staining, and ++++ , which signified intense staining of PAI-1. Hepatocytes, fibrous tissue, and tumour cells, where appropriate, were graded separately.

Monoclonal antibody to neurofilament protein, rabbit antibody to mouse polyclonal antibody, and the APAAP complex antibodies were obtained from Dakopatts, High Wycombe, Buckinghamshire.

\section{Results}

PAI-1 antigen was abundant in normal human liver. APAAP staining showed it to be present in discrete granules in the cytoplasm of hepatocytes particularly around portal tracts and central veins in the liver lobule (fig 1A). PAI-1 was predictably found in vascular endothelium and was most obvious in arteries and veins that run in the portal tracts. PAI-1 was also found in the biliary epithelium, in connective tissue of blood vessels, and in Glisson's capsule of the liver surface. No colour development was seen with the control antibody (fig 1B).

In the 10 cases of hepatic cirrhosis stained using the APAAP technique most showed a dramatic reduction in PAI-1 content of liver parenchyma compared with the normal liver sections (fig 2). Variable PAI-1 staining was found in the fibrous tissue in cirrhotic liver, as in the connective tissue of normal liver.

Sections of liver affected by primary biliary cirrhosis showed a similar lack of PAI-1 staining, with a variable amount of staining of fibrous tissue (not shown).

Livers with metastases showed a variable amount of PAI-1 staining, some with slightly more than the normal, whereas others displayed a considerable increase above normal. In these the PAI-1 staining was most intense immediately adjacent to the tumour margin. The granules of PAI-1 were more densely packed in the hepatocytes and present in a higher proportion of hepatocytes (fig 3).

In contrast, non-cirrhotic liver affected by primary hepatocellular carcinoma contained PAI-1 in similar quantities to those found in the normal liver sections. In one case the primary carcinoma was superimposed on cirrhosis and showed very little PAI-1 in the hepatocytes, which was consistent with the findings in cirrhosis alone (not shown).

Primary and secondary carcinoma cells infiltrating liver showed minimal PAI-1 staining. Fibrous tissue did contain some PAI-1, as in normal and cirrhotic liver sections.

The table shows the grading for specific PAI-1 staining for normal and diseased liver sections. temperature. Developing solution consisted of $0.05 \%(w / v)$ napthol AS-MX phosphate $0.012 \%(\mathrm{w} / \mathrm{v})$, levamisole, and $0.05 \%(\mathrm{w} / \mathrm{v})$ Fast Red TR salt, each dissolved in veronal acetate buffer (29 $\mathrm{mM}$ barbitone, $29 \mathrm{mM}$ 


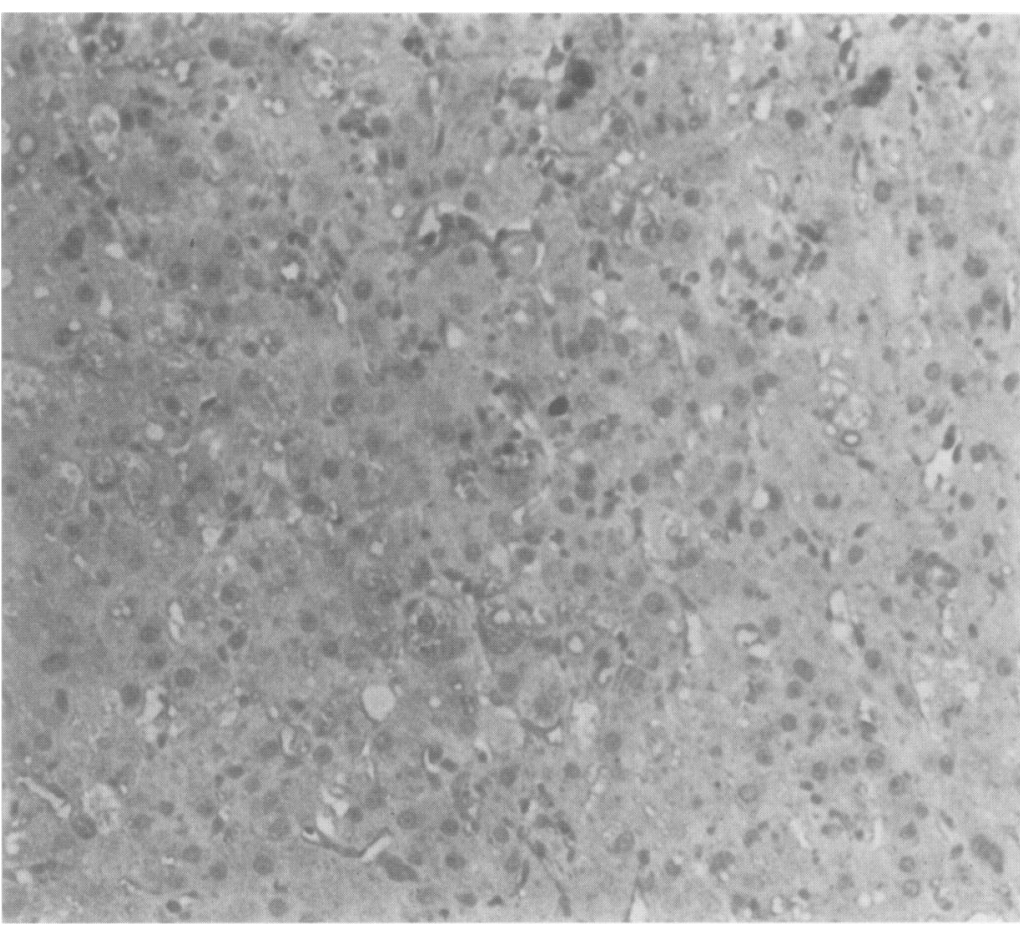

Figure 2 Cirrhotic liver section stained with monoclonal antibody to PAI-1.

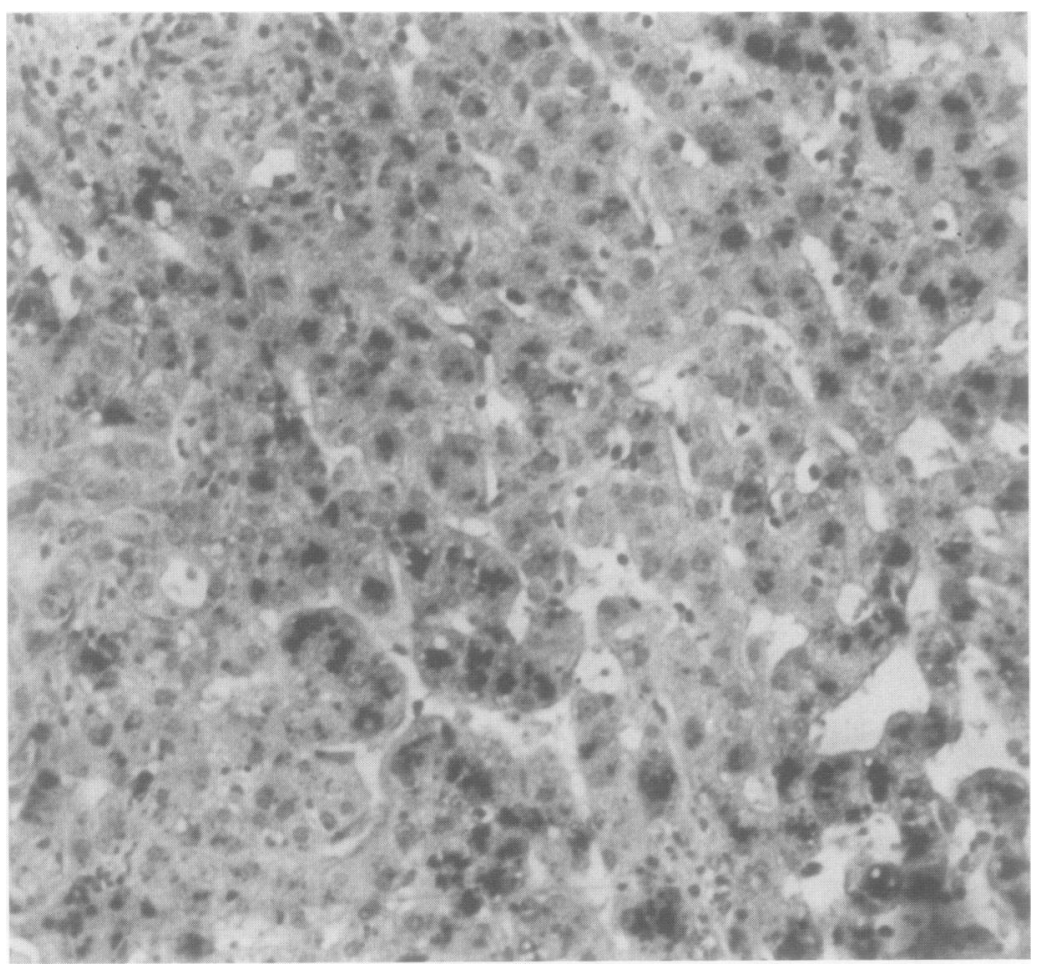

Figure 3 Liver section containing metastatic carcinoma stained with monoclonal antibody to PAI-1.

Grading of PAI-1 staining in normal liver, cirrhotic liver, and liver with metastases

\begin{tabular}{lll}
\hline Normal liver & Cirrhotic liver & Liver with metastases \\
\hline++ & + & ++ \\
++ & + & +++ \\
++ & + & +++ \\
++ & \pm & ++ \\
& + & ++ \\
& + & +++ \\
& + & ++ \\
& + & \\
& ++ & \\
& + & \\
& + & \\
& ++ & \\
& &
\end{tabular}

$\pm=$ Traces only

$++++=$ Dense staining

\section{Discussion}

t-PA is cleared by the liver and PAI-1 participates in the process. ${ }^{23} 23$ PAI-1 may be synthesised in the liver, ${ }^{10} 24$ but whether it is this PAI-1 that takes part in clearance is unknown. We found PAI-1 antigen in cytoplasmic granules, particularly in the centrilobular area and around the portal tracts of the liver lobule. In the centrilobular area the PAI-1 granules were of fairly uniform size and seemed to colocalise with lipofuscin. This suggests that the PAI-1 here had been cleared from the circulation and shifted into lysosomes for degradation. Around the portal tracts these granules were more variable in size and could be PAI-1 in the Golgi apparatus.

We found that cirrhotic liver contains considerably less PAI-1 antigen than normal liver. There are three possible explanations for this: (a) the synthesis of PAI-1 is reduced; $(b)$ the release of PAI-1 from hepatocytes is increased; and (c) the clearance of PAI-1 from the circulation is reduced. This study cannot distinguish which combination of these possibilities takes place but the second and third mechanisms seem likely. PAI-1 concentrations in the plasma are increased above normal in cirrhotic patients, ${ }^{171825}$ so excess release may be contributing to the reduced amounts of PAI-1 antigen in cirrhotic liver parenchyma; in cirrhotic patients vascular endothelium outside the liver may also be releasing more PAI-1 into the circulation. The increased plasma concentration of t-PA-PAI-1 complex in cirrhosis, together with the reduced PAI-1 antigen in cirrhotic liver, suggests that hepatic receptor activity for t-PA-PAI-1 complex is reduced in cirrhosis.

APAAP staining of liver with metastases showed that PAI-1 was often increased in the normal parenchyma surrounding the tumour. This was particularly intense immediately adjacent to the tumour margin and seemed to diminish further away from the margin. This suggests that clearance of PAI-1 is not the mechanism producing this pattern; it seems more likely that either the tumour itself is producing PAI-1 and releasing it locally or that the tumour stimulates the normal parenchyma to produce more PAI-1. The absence of staining in tumour cells suggests that the tumour stimulates the production. If it is correct this could represent a physiological mechanism by which the liver responds to local spread of the tumour. In support of this Kircheimer et al have shown that plasma urokinase activity is increased in hepatic malignancy and that this is likely to be due to tumour growth rather than reduced clearance of u-PA ${ }^{26}$; local PAI-1 synthesis might represent the hepatic response to this. Both transforming growth factor $\beta$ and epidermal growth factor have also been shown to increase in vitro production of PAI-1 from Hep G2 cells. ${ }^{1127}$ Patients with metastatic carcinoma are prone to venous thrombosis and have various haemostatic abnormalities, including fibrinolytic abnormalities with raised plasma concentrations of PAI- $1 .{ }^{18} 19$ Our study provides evidence that increased 
PAI-1 content in the liver of these patients stimulated by the presence of tumour might be a mechanism contributing to these clinical features. Local synthesis of PAI-1 might therefore influence tumour spread within the liver and contribute to the tendency for the thrombosis seen in malignancy.

Lastly, the virtual absence of PAI-1 from tumour cells of primary hepatocellular carcinoma suggests that hepatoma cell lines may not be ideal for studying physiological regulation of PAI-1 in normal human liver.

1 Bennett B, Croll A, Ferguson K, Booth NA. Complexing of tissue plasminogen activator with PAI-1, alpha-2 macroglobulin and C1-inhibitor: studies in patients with defibrination and a fibrinolytic state after electroshock or complicated labour. Blood 1990;75:671-6.

2 Wing LR, Hawksworth GM, Bennett B, Booth NA Clearance of t-PA, PAI-1 and t-PA-PAI-1 complex in an isolated perfused rat liver system. Fournal of Clinical Medicine 1991;117:109-14

3 Wing L, Bennett B, Booth NA. The receptor for tissue plasminogen activator ( $t-P A$ ) in complex with its inhibitor, PAI-1, on human hepatocytes. FEBS 1991; 278:95-7.

4 Smedsrod B, Einarson M, Pertoft $H$. Tissue plasminogen activator endocytosed by mannose and galactose recep tors of rat liver cells. Thromb Haemost 1988;59:480-4.

5 Smedsrod B, Einarson M. Clearance of tissue plasminogen activator by mannose and galactose receptors in the liver. Thromb Haemost 1990;63:60-6.

6 Marks GJ, Hart TK, Rush GF, Hoffstein ST, Fong K-LL, Bugesski PJ. Internalization of recombinant tissue-type plasminogen activator by isolated hepatocytes is via coated pits. Thromb Haemost 1990;63:251.

7 Einarson M, Haggroth L, Mattsson C. Elimination of native and carbohydrate modified tissue plasminogen native and carbohydrate modified tissue plasmin
activator in rabbits. Thromb Haemost 1989;64:1088.

8 Tanswell P, Schlutter M, Krause J. Pharmacolinetics and isolated liver perfusion of carbohydrate modified recombinant tissue-type plasminogen activator. Fibrinolysi 1989;3:79-84.

9 Simpson AJ, Booth NA, Moore NR, Bennett B. Distribution plasminogen activator inhibitor (PAI-1) in tissues. $\mathcal{F}$ Clin Pathol 1991;44:139-43.

10 Sprengers ED, Princen HMG, Kooistra T, Van Hinsbergh $V$. Inhibition of plasminogen activators by conditioned medium of human hepatocytes and hepatoma cell line, Hep G2. $₹$ Lab Clin Med 1985;105:751-8.

11 Lucore CL, Fujii S, Wun T-C, Sobel BE, Billadello JJ.
Regulation of the expression of type I plasminogen activator inhibitor in Hep $\mathrm{G} 2$ cells by epidermal growth factor. $\mathcal{F}$ Biol Chem 1988;263:15845-8.

12 Schneiderman J, Sawdey M, Loskutoff DJ. Type I plasminogen activator (PAI-1) gene expression in regenerating liver. Fibrinolysis 1992;6(suppl 2):19.

13 Thornton AJ, Bruzdzinski CJ, Raper SE, Gelehter TD. Plasminogen activator inhibitor 1 is an immediate early Plasminogen activator inhibitor 1 is an immediate early response gene expressed during
Fibrinolysis, 1992;6(suppl 2):19.

14 de Nicola P, Soardi F. Fibrinolysis in liver diseases: study of cases by means of the fibrin plate method. Thrombosis et Diathesis Haemorrhagica 1958;2:290-9.

15 Fletcher AP, Biederman O, Moore D, Alkjaersig N, Sherry $\mathrm{S}$. Abnormal plasminogen-plasmin system activity (fibrinolysis) in patients with hepatic cirrhosis: its cause and consequences. F Clin Invest 1964;43:681-95.

16 Booth NA, Anderson JA, Bennett B. Plasminogen activators in alcoholic cirrhosis: demonstration of increased tissue-type and urokinase-type activator. $f$ Clin Pathol tissue-type and

17 Tran-Thang C, Fasel-Kelly J, Pralong G, Hofstetter J-R Bachmann F, Kruithof EKO. Plasminogen activator and plasminogen activator inhibitors in liver deficiencies caused by chronic alcoholism or infectious hepatitis. Thromb Haemost, 1989;62:651-3.

18 Leiper K, Croll A, Booth NA, Moore NR, Sinclair T, Bennett B. Tissue plasminogen activator, plasminogen activator inhibitors, activation-inhibitor complex in liver disease. $f$ Clin Pathol 1994;47:214-7.

19 Nand S, Messmore H. Hemostasis and malignancy. $A m \mathcal{F}$ Hematol 1990;35:45-55.

$20 \mathrm{Kwaan}$ HC, Lo R, McFadzean AJS. Antifibrinolytic activity in primary carcinoma of the liver. Clin Sci 1959;18: in primary

21 Cordell $\mathrm{L}$, Falinia B, Erber WN, et al. Immunoenzymatic labelling of monoclonal antibodies using immune complexes of alkaline phosphatase and monoclonal anti-alkaline phosphatase (APAAP complexes). 7 Histochem Cytochem 1984;32:219-29.

22 MacGregor IR, Tonner AM, Micklem LR, James K, Booth NA. Murine monoclonal antibodies against active site epitopes on human endothelial plasminogen activator inhibitor (PAI-1). Fibrinolysis 1990;4:27-34.

23 Emeis J. Fast hepatic clearance of tissue type plasminogen activator inhibitor. Thromb Haemost 1985;54:230.

24 Fujii S, Lucore CL, Hopkins WE, Billadello JJ, Sobel BE Induction of synthesis in human hepatic and endothelia cells. Thromb Haemost 1990;64:412-9.

25 Lasierra J, Aza MJ, Vilades E, Poblet S, Barrao F, Bayon $\mathrm{E}$, Gonzalez J. Tissue plasminogen activator and plasminogen activator inhibitor in patients with liver cirrhosis. Fibrinolysis 1991;5:117-20.

26 Kircheimer JC, Huber K, Polterauer P, Binder BR. Urokinase antigen in plasma of patients with liver cirrhosis and hepatoma. Thromb Haemost 1985;54:617-8.

27 Fujii S, Lucore CL, Hopkins WE, Billadello JJ, Sobel BE Potential attenuation of fibrinolysis by growth factors released from platelets and their pharmacologic implications. Am $\mathcal{F}$ Cardiol 1989;63:1505-11. 